TRANSACTIONS OF THE

AMERICAN MATHEMATICAL SOCIETY

Volume 357, Number 9, Pages 3509-3523

S 0002-9947(04)03584-6

Article electronically published on October 7, 2004

\title{
TANGENT ALGEBRAIC SUBVARIETIES OF VECTOR FIELDS
}

\author{
JUAN B. SANCHO DE SALAS
}

\begin{abstract}
An algebraic commutative group $G$ is associated to any vector field $D$ on a complete algebraic variety $X$. The group $G$ acts on $X$ and its orbits are the minimal subvarieties of $X$ which are tangent to $D$. This group is computed in the case of a vector field on $\mathbb{P}_{n}$.
\end{abstract}

\section{INTRODUCTION}

Let $X$ be an algebraic variety over an algebraically closed field $k$ of characteristic 0 and let $D$ be a vector field on $X$. A closed subvariety $Y$ of $X$, defined by a sheaf of ideals $I$ of $\mathcal{O}_{X}$, is said to be a tangent subvariety of $D$ if $D(I) \subseteq I$. This condition implies that $D$ induces a derivation of the sheaf $\mathcal{O}_{Y}=\mathcal{O}_{X} / I$, so that it defines a vector field on $Y$. The aim of this paper is to study the structure of the family of tangent subvarieties of a vector field $D$. At first sight it seems reasonable to conjecture that minimal tangent subvarieties of $D$ form an algebraic family. Precisely:

Question: Does there exist a dense open set $U$ in $X$ and a surjective morphism of $k$-schemes $\pi: U \rightarrow Z$ whose fibres are just the minimal tangent subvarieties of $D$ in $U$ ?

Unfortunately, the answer is negative in general (we shall give a counterexample in Section 5). However, it is affirmative in an interesting case, that of global vector fields on a complete algebraic variety. For these fields we prove the following result:

Theorem. Let $D$ be a vector field on an integral projective variety $X$. There exists a connected commutative algebraic subgroup $G \subseteq$ Aut $X$ and a $G$-invariant dense open set $U$ in $X$ such that:

a) Minimal tangent subvarieties of $D$ on $U$ are just orbits of $G$ in $U$.

b) The quotient variety $\pi: U \rightarrow Z=U / G$ exists. Hence, the fibres of $\pi$ are the minimal tangent subvarieties of $D$ in $U$.

c) The sheaf $\mathcal{O}_{Z}$ coincides with the sheaf of first integrals of $D$; that is to say, for any open set $V$ in $Z$, we have

$$
\Gamma\left(V, \mathcal{O}_{Z}\right)=\left\{f \in \Gamma\left(\pi^{-1} V, \mathcal{O}_{X}\right): D f=0\right\} .
$$

This theorem may be extended to a distribution on $X$ generated by global vector fields (see 3.6).

The completeness of $X$ is used to assure the existence of the algebraic group Aut $X$. In such a case, the Lie algebra of all vector fields to $X$ is canonically isomorphic to the Lie algebra of the group Aut $X$. Hence, the vector field $D$

Received by the editors February 14, 2003 and, in revised form, November 19, 2003.

2000 Mathematics Subject Classification. Primary 14L30.

(C)2004 American Mathematical Society 
corresponds to some element $\tilde{D}$ in the Lie algebra of Aut $X$, that is to say, $D$ is a fundamental vector field with respect to the action of Aut $X$ on $X$. In fact, the former theorem holds when $X$ is not complete, if $D$ is assumed to be a fundamental field with respect to the action on $X$ of some algebraic group.

The above mentioned commutative group $G$ is defined to be the minimal tangent subvariety of $\tilde{D}$ through the identity of Aut $X$.

We determine the group $G$ in the case of vector fields on the complex projective space $\mathbb{P}_{n}$. It is well known that any vector field on $\mathbb{P}_{n}$ may be expressed as $D=$ $\pi_{*}\left(\sum \lambda_{i j} z_{j} \frac{\partial}{\partial z_{i}}\right)$, where $\pi: \mathbb{C}^{n+1} \rightarrow \mathbb{P}_{n}$ is the natural projection. The group $G$ is determined by two integers $s, \delta$ associated to $D$ as follows. Let us consider the field $\mathbb{C}$ as an infinite dimensional affine space over $\mathbb{Q}$; then $s$ is the dimension of the minimal affine $\mathbb{Q}$-subspace of $\mathbb{C}$ containing all the eigenvalues of the matrix $\left(\lambda_{i j}\right)$. The integer $\delta$ is 0 when the matrix $\left(\lambda_{i j}\right)$ is diagonalizable and 1 otherwise. We prove the following result:

Theorem. The group $G$ associated to a vector field $D=\pi_{*}\left(\sum \lambda_{i j} z_{j} \frac{\partial}{\partial z_{i}}\right)$ on the complex projective space $\mathbb{P}_{n}$ is

$$
G=\left(\mathbb{G}_{m}\right)^{s} \times\left(\mathbb{G}_{a}\right)^{\delta} .
$$

( $\mathbb{G}_{m}$ and $\mathbb{G}_{a}$ are the multiplicative and additive lines respectively.)

Corollary. The dimension of the minimal tangent subvarieties of $D$ is $s+\delta$ (generically). The field of meromorphic functions on $\mathbb{P}_{n}$ which are first integrals of $D$ has transcendence degree equal to $n-s-\delta$.

\section{Preliminaries}

From now on, $k$ will be an algebraically closed field of characteristic 0 and $X$ will be a $k$-scheme.

1.1. Differential algebras. Let us recall some elementary facts about differential algebras. A differential $k$-algebra is a $k$-algebra $A$ endowed with a $k$-linear derivation $D: A \rightarrow A$. An ideal $I$ of $A$ is said to be a differential ideal if $D(I) \subseteq I$.

Proposition 1.1. The nilradical of any differential algebra $A$ is a differential ideal.

Proof. If $a^{n}=0$, applying $D^{n}$ we obtain $n !(D a)^{n}+b a=0$, so that $D a$ is nilpotent.

Proposition 1.2. Any minimal prime ideal of a differential noetherian $k$-algebra is a differential ideal.

Proof. Replacing $A$ by $A / \operatorname{rad} A$, we may assume that $\operatorname{rad} A=0$. In such case, $0=\mathfrak{p}_{1} \cap \cdots \cap \mathfrak{p}_{r}$ where $\mathfrak{p}_{1}, \ldots, \mathfrak{p}_{r}$ are the minimal prime ideals of $A$. Let $0 \neq b \in$ $\mathfrak{p}_{2} \cap \cdots \cap \mathfrak{p}_{r}$. If $a \in \mathfrak{p}_{1}$, then $a b=0$ because $a b \in \mathfrak{p}_{1} \cap \cdots \cap \mathfrak{p}_{r}=0$. Hence $(D a) b=$ $-a D b$, so that both terms are 0 , since $a D b \in \mathfrak{p}_{1}$ and $b D a \in \mathfrak{p}_{2} \cap \cdots \cap \mathfrak{p}_{r}$. Finally, from $(D a) b=0$ it follows that $D a \in \mathfrak{p}_{1}$ because $b \notin \mathfrak{p}_{1}$. Therefore $D \mathfrak{p}_{1} \subseteq \mathfrak{p}_{1}$. 
1.2. Functor of points. The functor of points of $X$ is defined to be the following contravariant functor on the category of $k$-schemes

$$
X^{\bullet}(T):=\operatorname{Hom}_{k}(T, X) .
$$

For any two $k$-schemes $X, Y$ we have (Yoneda's lemma)

$$
\operatorname{Hom}_{k}(X, Y)=\operatorname{Hom}_{\text {funct. }}\left(X^{\bullet}, Y^{\bullet}\right) \text {. }
$$

In particular, in order to define a morphism of $k$-schemes $X \rightarrow Y$ it is enough to construct a morphism of functors $X^{\bullet} \rightarrow Y^{\bullet}$. We shall use this fact later on.

Elements of $X^{\bullet}(T)$, that is to say, morphisms of $k$-schemes $x: T \rightarrow X$, are said to be $T$-valued points (or $T$-points) of $X$. Moreover, we use the following convention: Given a $T$-point $x: T \rightarrow X$ and a morphism of $k$-schemes $T^{\prime} \rightarrow T$, the composition $T^{\prime} \longrightarrow T \stackrel{x}{\longrightarrow} X$ will be denoted also by $x$, that is to say, we do not change the notation of a point after a basis change.

1.3. Infinitesimal automorphisms. Let $k[\varepsilon]=k[x] /\left(x^{2}\right)$ and let $i: X \hookrightarrow X_{k[\varepsilon]}=$ $X \times_{k} k[\varepsilon]$ be the closed immersion defined by $\varepsilon=0$.

An infinitesimal automorphism of $X$ is defined to be an automorphism of $k[\varepsilon]$-schemes $\tau_{\varepsilon}: X_{k[\varepsilon]} \rightarrow X_{k[\varepsilon]}$ satisfying the infinitesimal condition

$$
\left(\tau_{\varepsilon}\right)_{\mid \varepsilon=0}=\operatorname{id}_{X}
$$

that is to say, $\tau_{\varepsilon} \circ i=i$.

Any vector field $D$ on $X$ determines an infinitesimal automorphism $\tau_{\varepsilon}$ of $X$ given by the following morphism of $k[\varepsilon]$-algebras:

$$
\tau_{\varepsilon}: \mathcal{O}_{X}[\varepsilon] \longrightarrow \mathcal{O}_{X}[\varepsilon], \quad a \mapsto \tau_{\varepsilon}(a)=a+\varepsilon D a
$$

and, conversely, any infinitesimal automorphism is clearly defined by a unique vector field.

1.4. Tangent subschemes. Let $D$ be a vector field on $X$. A closed subscheme $Y$ of $X$, defined by a sheaf of ideals $I$ of $\mathcal{O}_{X}$, is said to be a tangent subscheme of $D$ if $D(I) \subseteq I$. This condition implies that $D$ induces a derivation of the sheaf $\mathcal{O}_{Y}=\mathcal{O}_{X} / I$, so that it defines a vector field on $Y$.

A closed subscheme $Y$ of $X$ is a tangent subscheme of $D$ if and only if its functor of points $Y^{\bullet}$ is stable under the corresponding infinitesimal automorphism $\tau_{\varepsilon}$, that is to say, for any $k$-scheme $T$ and any point $x \in X^{\bullet}(T)$, we have

$$
x \in Y^{\bullet}(T) \Rightarrow \tau_{\varepsilon}(x) \in Y_{k[\varepsilon]}^{\bullet}\left(T_{k[\varepsilon]}\right)=Y^{\bullet}\left(T_{k[\varepsilon]}\right)
$$

where $\tau_{\varepsilon}(x)$ stands for the composition $T_{k[\varepsilon]} \stackrel{x}{\longrightarrow} X_{k[\varepsilon]} \stackrel{\tau_{\varepsilon}}{\longrightarrow} X_{k[\varepsilon]}$.

Given a closed point $x \in X$, we denote by $Y_{x}$ the minimal tangent subscheme of $D$ passing through $x$. Remark that $Y_{x^{\prime}} \subseteq Y_{x}$ whenever $x^{\prime} \in Y_{x}$.

From Propositions 1.1 and 1.2 it follows that $Y_{x}$ is reduced and irreducible.

1.5. Zeros of a vector field. Let $D$ be a vector field on $X$ and let us consider the natural morphism $\Omega_{X}^{1} \stackrel{D}{\longrightarrow} \mathcal{O}_{X}, \mathrm{~d} a \mapsto D a$. The image of this morphism is a certain sheaf of ideals $I$ of $\mathcal{O}_{X}$. The subscheme of zeros of $D$ is the closed subscheme $Z_{D}$ of $X$ defined by the sheaf of ideals $I$.

The subscheme of zeros $Z_{D}$ may be defined in terms of the infinitesimal automorphism $\tau_{\varepsilon}$ corresponding to the vector field $D$. In fact, the functor of $\tau_{\varepsilon}$-invariant points of $X$ is representable by the subscheme of zeros $Z_{D}$ as follows. 
Lemma 1.3. We have

$$
Z_{D}^{\bullet}(T)=\left\{x \in X^{\bullet}(T): \tau_{\varepsilon}(x)=x\right\}
$$

where the equality $\tau_{\varepsilon}(x)=x$ states the coincidence of the composition morphism $T_{k[\varepsilon]} \stackrel{x}{\longrightarrow} X_{k[\varepsilon]} \stackrel{\tau_{\varepsilon}}{\longrightarrow} X_{k[\varepsilon]}$ with the morphism $T_{k[\varepsilon]} \stackrel{x}{\longrightarrow} X_{k[\varepsilon]}$.

Proof. A morphism $x: T \rightarrow X$ factors through $Z_{D}$ if and only if the composition $\Omega_{X}^{1} \stackrel{D}{\longrightarrow} \mathcal{O}_{X} \stackrel{x}{\longrightarrow} \mathcal{O}_{T}$ vanishes. Now, this condition is equivalent to the coincidence of the two following morphisms:

$$
\begin{array}{rlrl}
\mathcal{O}_{X}[\varepsilon] \stackrel{\tau_{\varepsilon}}{\longrightarrow} \mathcal{O}_{X}[\varepsilon] \stackrel{x}{\longrightarrow} \mathcal{O}_{T}[\varepsilon] & a \mapsto a+\varepsilon D a & \mapsto x(a)+\varepsilon x(D a), \\
\mathcal{O}_{X}[\varepsilon] \stackrel{\mathcal{O}_{T}}{\longrightarrow}[\varepsilon] & a \mapsto x(a) .
\end{array}
$$

1.6. Differential morphisms. Let $(A, D)$ and $\left(A^{\prime}, D^{\prime}\right)$ be two differential $k^{-}$ algebras. A morphism of $k$-algebras $\varphi: A \rightarrow A^{\prime}$ is said to be differential if it commutes with the given derivations: $\varphi(D a)=D^{\prime}(\varphi(a))$.

Now let $X$ and $X^{\prime}$ be two $k$-schemes and let $D$ and $D^{\prime}$ be vector fields on $X$ and $X^{\prime}$ respectively. A morphism of $k$-schemes $\varphi: X^{\prime} \rightarrow X$ is said to be differential when the morphism of $k$-algebras $\varphi: \mathcal{O}_{X}(U) \rightarrow \mathcal{O}_{X^{\prime}}(V)$ is differential, for any pair of open sets $U \subseteq X$ and $V \subseteq \varphi^{-1}(U)$.

Let $\tau_{\varepsilon}$ and $\tau_{\varepsilon}^{\prime}$ be the infinitesimal automorphisms corresponding to $D$ and $D^{\prime}$ respectively. A morphism of $k$-schemes $\varphi: X^{\prime} \rightarrow X$ is differential if and only if the morphism $\varphi: X_{k[\varepsilon]}^{\prime} \longrightarrow X_{k[\varepsilon]}$ satisfies $\varphi \circ \tau_{\varepsilon}^{\prime}=\tau_{\varepsilon} \circ \varphi$.

The following statement is trivial.

Proposition 1.4. Let $\varphi:\left(X^{\prime}, D^{\prime}\right) \longrightarrow(X, D)$ be a differential morphism of $k^{-}$ schemes. If $Y$ is a tangent subscheme of $(X, D)$, then $\varphi^{-1}(Y)=Y \times_{X} X^{\prime}$ is a tangent subscheme of $\left(X^{\prime}, D^{\prime}\right)$.

\section{Algebraic group associated to A VECTOR FIELD}

In this section $X$ will be a proper $k$-scheme.

Definition 2.1. Let us consider the functor of automorphisms of $X$, defined on the category of $k$-schemes,

$$
F(T):=\operatorname{Aut}_{T}\left(X \times_{k} T\right) .
$$

This functor is representable (see [9]) by an algebraic group Aut $X$, named scheme of automorphisms of $X(4])$, that is to say,

$$
(\text { Aut } X)^{\bullet}(T)=\operatorname{Aut}_{T}\left(X \times_{k} T\right) .
$$

Now let $D$ be a vector field on $X$ and let $\tau_{\varepsilon}$ be the corresponding infinitesimal automorphism. This automorphism induces an infinitesimal automorphism $\tilde{\tau}_{\varepsilon}$ of Aut $X$. We construct this automorphism $\tilde{\tau}_{\varepsilon}:(\text { Aut } X)_{k[\varepsilon]} \longrightarrow(\text { Aut } X)_{k[\varepsilon]}$ by means of the functor of points: For any $k[\varepsilon]$-scheme $T$ we define

$$
\tilde{\tau}_{\epsilon}: \operatorname{Aut}_{T}\left(X_{k[\varepsilon]} \times_{k[\varepsilon]} T\right) \longrightarrow \operatorname{Aut}_{T}\left(X_{k[\varepsilon]} \times_{k[\varepsilon]} T\right), \quad g \mapsto \tau_{\varepsilon} \circ g .
$$

Note that $\tilde{\tau}_{\varepsilon}$ satisfies the infinitesimal condition $\left(\tilde{\tau}_{\varepsilon}\right)_{\mid \varepsilon=0}=\operatorname{id}_{\text {Aut } X}$. 
The infinitesimal automorphism $\tilde{\tau}_{\varepsilon}$ corresponds to a certain tangent field $\tilde{D}$ on Aut $X$. Note that, by definition, $\tilde{\tau}_{\varepsilon}$ commutes with right translations in Aut $X$, so that the field $\tilde{D}$ is invariant under right translations.

Therefore, any vector field $D$ on a proper $k$-scheme $X$ has a canonically associated vector field $\tilde{D}$ on the scheme of automorphisms Aut $X$, which is invariant under right translations.

Moreover, it may be proved that the map $D \mapsto \tilde{D}$ defines an isomorphism of the Lie algebra of vector fields on $X$ onto the Lie algebra of the group Aut $X$ (we shall not use this fact).

Definition 2.2. Let $D$ be a vector field on a proper $k$-scheme $X$ and let $\tilde{D}$ be the corresponding vector field on the scheme of automorphisms Aut $X$, invariant under right translations. The associated group of $D$ is defined to be the minimal tangent subscheme $G$ of $\tilde{D}$ passing through the identity of Aut $X$. We shall prove that $G$ is a commutative algebraic subgroup of Aut $X$.

Proposition 2.3. $G$ is a connected algebraic closed subgroup of Aut $X$.

Proof. By definition $G$ is a closed subscheme of Aut $X$. Moreover, $G$ is integral (hence connected) by Propositions 1.1 and 1.2. Finally, we have to show that the product $G \times G \longrightarrow$ Aut $X$ factors through $G$ and that the inverse morphism Aut $X \rightarrow$ Aut $X, g \mapsto g^{-1}$ takes $G$ into itself. Since $G$ is reduced, it is enough to prove both statements in the case of closed points; that is to say, it is enough to show that $G^{\bullet}(k)$ is a subgroup of $(\text { Aut } X)^{\bullet}(k)$.

Let $g \in G^{\bullet}(k)$. Since $G$ is the minimal tangent subvariety of $\tilde{D}$ through the identity and $\tilde{D}$ is invariant under right translations, it follows that $G \cdot g$ is the minimal tangent subvariety of $\tilde{D}$ passing through $g$. Hence $G \cdot g \subseteq G$, because $G$ also passes through $g$. This inclusion is not strict, since otherwise, multiplying at right by $g^{n}$, we would obtain an infinite decreasing sequence of closed sets $G \cdot g^{n+1} \subset G \cdot g^{n}$, so contradicting the noetherian character of Aut $X$. Hence $G \cdot g=G$ and then $G^{\bullet}(k) \cdot g=G^{\bullet}(k)$ for any $g \in G^{\bullet}(k)$. It follows readily that $G^{\bullet}(k)$ is a group.

Proposition 2.4. $G$ is commutative.

Proof. Let us consider the infinitesimal automorphism

$$
\sigma:(\text { Aut } X)_{k[\varepsilon]} \longrightarrow(\text { Aut } X)_{k[\varepsilon]}
$$

defined, in terms of the functor of points, by the formula $\sigma(g)=\tau_{\varepsilon} \circ g \circ \tau_{\varepsilon}^{-1}$. This infinitesimal automorphism corresponds to a certain vector field on Aut $X$. Let $H$ be the corresponding closed subscheme of zeros. By Lemma 1.3 the functor of points of $H$ is

$$
H^{\bullet}(T)=\left\{g \in(\text { Aut } X)^{\bullet}(T): \sigma(g)=g\right\}=\left\{g \in(\text { Aut } X)^{\bullet}(T): \tau_{\varepsilon} \circ g=g \circ \tau_{\varepsilon}\right\} \text {. }
$$

Remark that $H^{\bullet}(T)$ is a subgroup of $(\text { Aut } X)^{\bullet}(T)$, hence $H$ is a closed algebraic subgroup of Aut $X$. Let us consider the center $C$ of $H$, which is a commutative closed subgroup. The functor of points of the center is

$$
C^{\bullet}(T)=\left\{c \in H^{\bullet}(T): c \circ h=h \circ c \text { for any } h \in H^{\bullet}\left(T^{\prime}\right) \text { and any } T^{\prime} \rightarrow T\right\}
$$

(we refer to [2], II, $\S 13.7$ for the existence of the center). Let us prove that $C$ is a tangent subscheme of $\tilde{D}$. According to [1.4 we have to show that the functor $C^{\bullet}$ is stable by the automorphism $\tilde{\tau}_{\varepsilon}$ : If $c \in C^{\bullet}(T)$, let us prove that 
$\tilde{\tau}_{\varepsilon}(c)=\tau_{\varepsilon} \circ c \in C^{\bullet}\left(T_{k[\varepsilon]}\right)$; in fact, for any morphism $T^{\prime} \rightarrow T_{k[\varepsilon]}$ and any point $h \in H^{\bullet}\left(T^{\prime}\right)$ we have

$$
\left(\tau_{\varepsilon} \circ c\right) \circ h=\tau_{\varepsilon} \circ(c \circ h)=\tau_{\varepsilon} \circ(h \circ c)=h \circ\left(\tau_{\varepsilon} \circ c\right) .
$$

Finally, $G$ is contained in $C$, because $C$ is a tangent subscheme of $\tilde{D}$ passing through the identity and $G$ is minimal. Since $C$ is commutative, so is $G$.

Note 2.5. A more general result than Proposition 2.4 was proved by Chevalley [1] in the realm of Lie algebras. In fact, if $L$ is a Lie subalgebra of the Lie algebra of an algebraic group and $L_{\text {alg }}$ stands for the minimal algebraic Lie subalgebra (in the sense that it is the Lie algebra of an algebraic subgroup) containing $L$, then [1], Chap. II, Th. 13, states that $[L, L]=\left[L_{\mathrm{alg}}, L_{\mathrm{alg}}\right]$; hence $L_{\mathrm{alg}}$ is abelian whenever $L$ is. In particular, if $L=\langle D\rangle$, then $L_{\text {alg }}$ is an abelian Lie subalgebra.

Let us consider the structure of the associated algebraic group $G$. According to the fundamental structure theorem of algebraic groups (see [12] or [13]), $G$ has an affine connected normal subgroup $N$ such that the quotient $A=G / N$ is an abelian variety. Moreover, any connected commutative affine group is a direct product of multiplicative and additive lines: $N=\mathbb{G}_{m}^{r} \times \mathbb{G}_{a}^{\delta}$. In conclusion, $G$ is an extension of an abelian variety by a direct product of multiplicative and additive lines:

$$
0 \longrightarrow \mathbb{G}_{m}^{r} \times \mathbb{G}_{a}^{\delta} \longrightarrow G \longrightarrow A \longrightarrow 0
$$

When this extension is trivial ( $G$ being the associated group), one may easily prove that $\delta \leq 1$.

\section{TANGEnt Subschemes}

Let us recall the notation of the former section: $X$ is a proper scheme over an algebraically closed field $k$ of characteristic zero, $D$ is a vector field on $X$ and $G$ is the associated group. Recall that $G$ is a subgroup of the group Aut $X$, so that we have an obvious action $\mu: G \times X \rightarrow X$.

Let $\tilde{D}$ be the right-invariant vector field on Aut $X$ induced by $D$, and let $\tilde{\tau}_{\varepsilon}$ be the corresponding infinitesimal automorphism: $\tilde{\tau}_{\varepsilon}(g)=\tau_{\varepsilon} \circ g$. Let us consider $\tilde{D}$ as a vector field on $G$ (it may be done because $G$ is a tangent subscheme of $\tilde{D}$ ). Then we may consider on $G \times X$ the vector field $(\tilde{D}, 0)$, the corresponding infinitesimal automorphism being $\left(\tilde{\tau}_{\varepsilon}, I d\right)$.

Lemma 3.1. The natural action $\mu: G \times X \rightarrow X$ is a differential morphism. As well, for any closed point $x \in X$ the morphism $\mu_{x}: G=G \times\{x\} \subset G \times X \stackrel{\mu}{\longrightarrow} X$ is also differential.

Proof. We have to show that the action $\mu$ commutes with the respective infinitesimal automorphisms: $\mu \circ\left(\tilde{\tau}_{\varepsilon}, I d\right)=\tau_{\varepsilon} \circ \mu$. We prove it by means of the functor of points: Let $(g, x) \in G^{\bullet}(T) \times X^{\bullet}(T)=(G \times X)^{\bullet}(T)$, where $T$ is a $k[\varepsilon]$-scheme; we have

$\left(\mu \circ\left(\tilde{\tau}_{\varepsilon}, I d\right)\right)(g, x)=\mu\left(\tau_{\varepsilon} \cdot g, x\right)=\left(\tau_{\varepsilon} \cdot g\right)(x)=\tau_{\varepsilon}(g(x))=\tau_{\varepsilon}(\mu(g, x))=\left(\tau_{\varepsilon} \circ \mu\right)(g, x)$.

The second statement may be proved in a similar way. 
Theorem 3.2. Let $D$ be a vector field on a proper $k$-scheme $X$ and let $G$ be the associated group. For any closed point $x \in X$, the minimal tangent subscheme $Y_{x}$ passing through $x$ coincides with the closure of the orbit of $x$, that is,

$$
Y_{x}=\overline{G \cdot x} .
$$

Proof. Let us consider the differential morphism $\mu_{x}: G \rightarrow X, g \mapsto g \cdot x$. By Proposition 1.4, $\mu_{x}^{-1}\left(Y_{x}\right)$ is a tangent subscheme of $\tilde{D}$, which contains the identity of $G$. Since $G$ is minimal, we conclude that $G \subseteq \mu_{x}^{-1}\left(Y_{x}\right)$, hence $\mu_{x}(G)=G \cdot x \subseteq Y_{x}$ and it follows that $\overline{G \cdot x} \subseteq Y_{x}$.

In order to show the reverse inclusion $Y_{x} \subseteq \overline{G \cdot x}$, it is enough to show that $\overline{G \cdot x}$ is a tangent subscheme of $D$, because $Y_{x}$ is minimal. Let $g_{0}$ be the generic point of $G$ and let $x_{0}=\mu_{x}\left(g_{0}\right)$. It is clear that $x_{0}$ is the generic point of $\overline{G \cdot x}=\overline{\mu_{x}(G)}$. Let $\mathfrak{p}_{x_{0}}$ be the maximal ideal of $\mathcal{O}_{X, x_{0}}$. The exact sequence

$$
0 \longrightarrow \mathfrak{p}_{x_{0}} \longrightarrow \mathcal{O}_{X, x_{0}} \stackrel{\mu_{x}}{\longrightarrow} \mathcal{O}_{G, g_{0}},
$$

where the last morphism is differential, shows that $\mathfrak{p}_{x_{0}}$ is a differential ideal of $\mathcal{O}_{X, x_{0}}$. Let $\mathfrak{p}$ be the sheaf of ideals of the closed subscheme $\overline{G \cdot x}$. Since $x_{0}$ is the generic point of this subscheme, for any open set $U$ in $X$ we have

$$
\mathfrak{p}(U)=\mathcal{O}_{X}(U) \cap \mathfrak{p}_{x_{0}}
$$

(rigorously, $\mathfrak{p}=h^{-1}\left(\mathfrak{p}_{x_{0}}\right)$ where $h: \mathcal{O}_{X}(U) \rightarrow \mathcal{O}_{X, x_{0}}$ is the natural morphism). It readily follows that $\mathfrak{p}(U)$ is a differential ideal of $\mathcal{O}_{X}(U)$.

Proposition 3.3. Let $G \times X \rightarrow X$ be an action of a connected algebraic group $G$ over an integral quasi-projective $k$-scheme $X$. There is a $G$-invariant dense open set $U$ in $X$ such that the geometric quotient $\pi: U \rightarrow U / G$ exists.

We shall prove this result in the Appendix. Putting Theorem[3.2 and Proposition 3.3 together we obtain the following result.

Theorem 3.4. Let $D$ be a vector field on an integral projective $k$-scheme $X$ and let $G$ be the associated group. There exists a dense open set $U$ in $X$ such that:

a) $U$ is $G$-invariant and orbits of closed points in $U$ are just minimal tangent subvarieties of the vector field $D$ on $U$.

b) The geometric quotient $\pi: U \rightarrow Z=U / G$ exists. The sheaf $\mathcal{O}_{Z}$ coincides with the sheaf of first integrals of $D$, that is, for any open set $V$ in $Z$ we have

$$
\Gamma\left(V, \mathcal{O}_{Z}\right)=\left\{f \in \Gamma\left(\pi^{-1} V, \mathcal{O}_{X}\right): D f=0\right\} .
$$

Proof. a) Let $U$ be the open set whose existence states Proposition 3.3. The orbit of any closed point in $U$ is a closed subset of $U$, since it is the fibre of $\pi: U \rightarrow U / G$ over a closed point of $U / G$. Applying Theorem 3.2 to the field $D$ on $U$, we conclude that such orbits are just the minimal tangent subvarieties.

b) Since $\pi: U \rightarrow Z=U / G$ is a geometric quotient, the sheaf $\mathcal{O}_{Z}$ coincides with the sheaf of $G$-invariant functions,

$$
\Gamma\left(V, \mathcal{O}_{Z}\right)=\Gamma\left(\pi^{-1} V, \mathcal{O}_{X}\right)^{G},
$$

so that we have to show that a function $f \in \Gamma\left(\pi^{-1} V, \mathcal{O}_{X}\right)$ is $G$-invariant if and only if $D f=0$.

Given a closed point $x \in \pi^{-1} V$, let us consider the minimal tangent subvariety $Y_{x}=G \cdot x$ of $D$ passing through $x$. If $f$ is $G$-invariant, then $\left.f\right|_{Y_{x}}$ is constant, so that $(D f)(x)=\left(D\left(\left.f\right|_{Y_{x}}\right)\right)(x)=0$. 
Conversely, if $D f=0$, then we consider the ideal $I=(f-\lambda)$, where $\lambda:=f(x)$. It is a differential ideal, hence it defines a tangent closed subscheme $T$ of $D$. Since $x \in T$ and $Y_{x}$ is minimal, we have $Y_{x}=G \cdot x \subseteq T$, hence $f=\lambda$ on $Y_{x}=G \cdot x$. Therefore $f$ is constant on the orbits and we conclude that $f$ is $G$-invariant.

Remark 3.5. The above theorem holds when $X$ is not complete if $D$ is assumed to be a fundamental vector field with respect to the action $\mu: \mathcal{G} \times X \rightarrow X$ of some algebraic group $\mathcal{G}$, i.e, $D=\mu_{*}(\tilde{D}, 0)$ for some right-invariant vector field $\tilde{D}$ on $\mathcal{G}$. Recalling Definition 2.2 the associated group $G$ to the vector field $D$ is defined to be the minimal tangent subvariety of $\tilde{D}$ passing through the identity of $\mathcal{G}$. The results of Sections 2 and 3 remain valid, so that the associated group $G$ is a commutative connected algebraic subgroup of $\mathcal{G}$, and the minimal tangent subvarieties of $D$ are the closure of the orbits of $G$ on $X$.

Remark 3.6. Theorem 3.4 may be generalized to a distribution generated by global vector fields. Let $X$ be an integral projective $k$-scheme and let $L$ be a vector subspace of the space of all global vector fields on $X$.

A closed subscheme of $X$ is said to be a tangent subscheme of $L$ if it is a tangent subscheme of any $D \in L$. Recall that each vector field $D$ on $X$ corresponds with a right-invariant vector field $\tilde{D}$ on Aut $X$. Let $\tilde{L}$ be the space of all rightinvariant vector fields $\tilde{D}$ on Aut $X$ such that $D \in L$.

The results of Sections 2 and 3 (and their proofs) may be extended for the distribution $L$ :

1. The minimal tangent subscheme of $\tilde{L}$, passing through the identity of Aut $X$, is a connected closed algebraic subgroup $G$ of Aut $X$.

2. If $L$ is an abelian Lie algebra, then $G$ is a commutative group.

3. The minimal tangent subscheme $Y_{x}$ of $L$ passing through a closed point $x \in X$ is the closure of the orbit, $Y_{x}=\overline{G \cdot x}$.

4. There exists a dense $G$-invariant open subset $U$ in $X$ such that:

a) Orbits of closed points in $U$ are just minimal tangent subvarieties of $L$ on $U$;

b) The geometric quotient $\pi: U \rightarrow Z=U / G$ exists and the sheaf $\mathcal{O}_{Z}$ coincides with the sheaf of first integrals of $L$, that is, for any open set $V$ in $Z$ we have

$$
\Gamma\left(V, \mathcal{O}_{Z}\right)=\left\{f \in \Gamma\left(\pi^{-1} V, \mathcal{O}_{X}\right): D f=0 \text { for any } D \in L\right\}
$$

\section{Vector fields on $\mathbb{P}_{n}$}

The aim of this section is to calculate the associated algebraic group of any vector field on the complex projective space $\mathbb{P}_{n}$.

Lemma 4.1. Let us consider the differential algebras

$$
\begin{aligned}
& \mathbb{C}\left[z_{1}, \ldots, z_{r}\right], D=\sum \mu_{i} z_{i} \frac{\partial}{\partial z_{i}}, \\
& \mathbb{C}\left[z_{0}, z_{1}, \ldots, z_{r}\right], D=\frac{\partial}{\partial z_{0}}+\sum_{i>0} \mu_{i} z_{i} \frac{\partial}{\partial z_{i}}
\end{aligned}
$$

where $\mu_{1}, \ldots, \mu_{r} \in \mathbb{C}$. If $\mu_{1}, \ldots, \mu_{r}$ are linearly independent over $\mathbb{Q}$, then any non-null differential ideal of these algebras contains some monomial $z_{1}^{a_{1}} \cdots z_{r}^{a_{r}}$. 
Proof. The same argument holds in both algebras. It is easy to check that monomials $\lambda z_{1}^{a_{1}} \cdots z_{r}^{a_{r}}$ are the only eigenvectors of $D$. Let $E_{n}$ be the vector subspace of all polynomials of degree $\leq n$ and note that $D\left(E_{n}\right) \subseteq E_{n}$ in both cases. Let $I$ be a non-null differential ideal. It is clear that $I \cap E_{n} \neq 0$ when $n \gg 0$. Since the dimension of $I \cap E_{n}$ is finite, the linear map $D: I \cap E_{n} \longrightarrow I \cap E_{n}$ has some eigenvector, hence $I$ contains some monomial.

Corollary 4.2. If $\mu_{1}, \ldots, \mu_{r}$ are linearly independent over $\mathbb{Q}$, then the differential algebras

$$
\begin{aligned}
& \mathbb{C}\left[z_{1}, \ldots, z_{r},\left(z_{1} \cdots z_{r}\right)^{-1}\right], D=\sum \mu_{i} z_{i} \frac{\partial}{\partial z_{i}} \\
& \mathbb{C}\left[z_{0}, z_{1}, \ldots, z_{r},\left(z_{1} \cdots z_{r}\right)^{-1}\right], D=\frac{\partial}{\partial z_{0}}+\sum_{i>0} \mu_{i} z_{i} \frac{\partial}{\partial z_{i}}
\end{aligned}
$$

have no non-trivial differential ideal.

Proposition 4.3. If $\mu_{1}, \ldots, \mu_{r} \in \mathbb{C}$ are linearly independent over $\mathbb{Q}$, then the following differential algebras of holomorphic functions on $\mathbb{C}$,

$$
\mathbb{C}\left[e^{\mu_{1} t}, \ldots, e^{\mu_{r} t}, e^{-\sum \mu_{i} t}\right], \quad \mathbb{C}\left[t, e^{\mu_{1} t}, \ldots, e^{\mu_{r} t}, e^{-\sum \mu_{i} t}\right]
$$

(endowed with the derivation $\frac{\partial}{\partial t}$ ) have no non-trivial differential ideal.

Proof. Let us consider the obvious differential epimorphisms

$$
\begin{gathered}
\mathbb{C}\left[z_{1}, \ldots, z_{r}, \frac{1}{z_{1} \cdots z_{r}}\right] \longrightarrow \mathbb{C}\left[e^{\mu_{1} t}, \ldots, e^{\mu_{r} t}, e^{-\sum \mu_{i} t}\right], \\
\mathbb{C}\left[z_{0}, z_{1}, \ldots, z_{r}, \frac{1}{z_{1} \cdots z_{r}}\right] \longrightarrow \mathbb{C}\left[t, e^{\mu_{1} t}, \ldots, e^{\mu_{r} t}, e^{-\sum \mu_{i} t}\right],
\end{gathered}
$$

where the algebras on the left are endowed with the derivations considered in Corollary 4.2. These epimorphisms are isomorphisms because both have null kernel by Corollary 4.2 .

Remark 4.4. By the last isomorphism in the former proof, the holomorphic functions $t, e^{\mu_{1} t}, \ldots, e^{\mu_{r} t}$ are algebraically independent whenever $\mu_{1}, \ldots, \mu_{r}$ are linearly independent over $\mathbb{Q}$.

4.5. Let $M=\left(\lambda_{i j}\right)$ be an $n \times n$ matrix with complex coefficients. Let us consider the linear vector field

$$
D=\sum \lambda_{i j} z_{j} \frac{\partial}{\partial z_{i}}
$$

on $\mathbb{C}^{n}$. The corresponding infinitesimal automorphism $\tau_{\varepsilon}$ of $\mathbb{C}^{n}$ is a $\mathbb{C}[\varepsilon]$-linear transformation with matrix $I d+\varepsilon M \equiv e^{\varepsilon M}$. This automorphism induces an infinitesimal automorphism $\tilde{\tau}_{\varepsilon}$ of the full linear group $G l_{n}$,

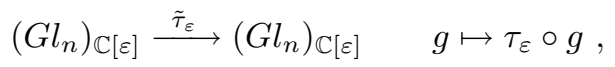

whose corresponding vector field $\tilde{D}$ on $G l_{n}$ is obviously right-invariant. Note that $D$ is a fundamental vector field with respect to the natural action $\mu: G l_{n} \times \mathbb{C}^{n} \rightarrow \mathbb{C}^{n}$, since we have $\mu_{*}(\tilde{D}, 0)=D$ because $\mu \circ\left(\tilde{\tau}_{\varepsilon}, I d\right)=\tau_{\varepsilon} \circ \mu$.

According to Remark 3.5, the associated group $G$ of the linear vector field $D$ is defined to be the minimal tangent subvariety of $\tilde{D}$ passing through the identity of $G l_{n}$. We know that $G$ is a commutative connected algebraic subgroup of $G l_{n}$, 
and that the minimal tangent subvarieties of $D$ are the closure of the orbits of $G$ on $\mathbb{C}^{n}$.

Indeed, it may be proved that the associated group $G$ of a linear vector field $D=\sum \lambda_{i j} z_{j} \frac{\partial}{\partial z_{i}}$ coincides with the so-called differential Galois group of the linear differential system $z_{i}(t)^{\prime}=\sum_{j} \lambda_{i j} z_{j}(t)$. See Note 4.7 below.

Let $\mathbb{C}\left[e^{M t}, \operatorname{det}\left(e^{-M t}\right)\right]$ be the algebra of holomorphic functions on $\mathbb{C}$ generated by the coefficients of the matrix $e^{M t}$ and the function $\operatorname{det}\left(e^{-M t}\right)$. It is a differential algebra with the derivation $\frac{\partial}{\partial t}$.

Lemma 4.6. The group $G$ associated to a linear vector field $D=\sum \lambda_{i j} z_{j} \frac{\partial}{\partial z_{i}}$ is

$$
G=\operatorname{Spec} \mathbb{C}\left[e^{M t}, \operatorname{det}\left(e^{-M t}\right)\right]
$$

where $M=\left(\lambda_{i j}\right)$.

Proof. Let $G l_{n}=\operatorname{Spec} \mathbb{C}\left[x_{i j}, \operatorname{det}\left(x_{i j}\right)^{-1}\right]$. The infinitesimal automorphism $\tilde{\tau}_{\varepsilon}$ is

$$
\tilde{\tau}_{\varepsilon}\left(\left(x_{i j}\right)\right)=\tau_{\varepsilon} \circ\left(x_{i j}\right)=e^{M \varepsilon} \circ\left(x_{i j}\right)=(I d+\varepsilon M) \circ\left(x_{i j}\right)=\left(x_{i j}\right)+\varepsilon M \circ\left(x_{i j}\right),
$$

so that the derivation $\tilde{D}$ is (in matrix form)

$$
\left(\tilde{D} x_{i j}\right)=M \circ\left(x_{i j}\right)
$$

Let us consider the obvious epimorphism

$$
\mathbb{C}\left[x_{i j}, \operatorname{det}\left(x_{i j}\right)^{-1}\right] \longrightarrow \mathbb{C}\left[e^{M t}, \operatorname{det}\left(e^{-M t}\right)\right]
$$

which transforms the coefficients of the universal matrix $\left(x_{i j}\right)$ into the coefficients of the matrix $e^{M t}$. Equality $(*)$ states that it is a differential morphism when the first algebra is endowed with the derivation $\tilde{D}$ and the second algebra is endowed with $\frac{\partial}{\partial t}$. Therefore Spec $\mathbb{C}\left[e^{M t}, \operatorname{det}\left(e^{-M t}\right)\right]$ is a tangent subscheme of $\tilde{D}$. Clearly, this closed subscheme passes through the identity of $G l_{n}$ when $t=0$. In order to show that it is the minimal tangent subscheme, it is enough to show that the differential algebra $\mathbb{C}\left[e^{M t}, \operatorname{det}\left(e^{-M t}\right)\right]$ has no non-trivial differential ideal.

After a linear coordinate change, we may assume that the matrix $M$ is in Jordan form. If $\lambda_{1}, \ldots, \lambda_{n}$ are the eigenvalues of $M$, then it is easy to check that

$$
\mathbb{C}\left[e^{M t}, \operatorname{det}\left(e^{-M t}\right)\right]=\mathbb{C}\left[\delta t, e^{\lambda_{1} t}, \ldots, e^{\lambda_{n} t}, e^{-\sum \lambda_{i} t}\right]
$$

where $\delta=0$ if $M$ is diagonalizable and $\delta=1$ otherwise.

Let $\mu_{1}, \ldots, \mu_{r}$ be a base of the $\mathbb{Q}$-vector space $\mathbb{Q} \lambda_{1}+\cdots+\mathbb{Q} \lambda_{n} \subset \mathbb{C}$, that is to say, $\mathbb{Q} \lambda_{1}+\cdots+\mathbb{Q} \lambda_{n}=\mathbb{Q} \mu_{1} \oplus \cdots \oplus \mathbb{Q} \mu_{r}$. It is easy to check that

$$
\mathbb{C}\left[\delta t, e^{\lambda_{1} t}, \ldots, e^{\lambda_{n} t}, e^{-\sum \lambda_{i} t}\right]=\mathbb{C}\left[\delta t, e^{\mu_{1} t}, \ldots, e^{\mu_{r} t}, e^{-\sum \mu_{j} t}\right] .
$$

Now, by Proposition 4.3 , we conclude that $\mathbb{C}\left[e^{M t}, \operatorname{det}\left(e^{-M t}\right)\right]$ has no non-trivial differential ideal.

Note 4.7. Let us explain the relation of the associated group $G$ with the Galois theory of differential equations.

We have shown in the former proof that $\mathbb{C}\left[e^{M t}, \operatorname{det}\left(e^{-M t}\right)\right]$ is a simple differential ring, i.e., it has no non-trivial differential ideal. According to (10, Def. 1.15), this ring is the Picard-Vessiot ring of the differential equation $z^{\prime}=M z$, since $e^{M}$ is a fundamental matrix. Let $L$ be the field of fractions of the Picard-Vessiot ring, 
which is named the Picard-Vessiot field of the equation $z^{\prime}=M z$ ([10], Def. 1.21). Now, by Lemma 4.6] $L$ is the field of functions of the closed algebraic subgroup $(G, \tilde{D}) \subset\left(G l_{n}, \tilde{D}\right)$. The action of $G$ on itself by right-translations induces an action of $G$ on $L$ by differential automorphisms. It is immediate to check that any $G$-invariant function is constant: $L^{G}=\mathbb{C}$. Then, by the Galois correspondence ([10], Prop. 1.34), we conclude that $G$ is the group of all differential automorphisms of $L$, i.e., $G$ is the differential Galois group of the equation $z^{\prime}=M z$.

The following theorem improves a statement of (8), Prop. 3.27), about the differential Galois group of a linear differential system with constant coefficients.

Theorem 4.8. The associated group of a linear vector field $D=\sum \lambda_{i j} z_{j} \frac{\partial}{\partial z_{i}}$ on $\mathbb{C}^{n}$ is

$$
G=\mathbb{G}_{m}^{r} \times \mathbb{G}_{a}^{\delta}
$$

where $r$ stands for the dimension of the $\mathbb{Q}$-vector space $\mathbb{Q} \lambda_{1}+\cdots+\mathbb{Q} \lambda_{n} \subset \mathbb{C}$ spanned by the eigenvalues of the matrix $M=\left(\lambda_{i j}\right)$, and $\delta=0$ when $M$ is diagonalizable and $\delta=1$ otherwise.

Proof. Again we put $G l_{n}=\operatorname{Spec} \mathbb{C}\left[x_{i j}, \operatorname{det}\left(x_{i j}\right)^{-1}\right]$. The group law in $G l_{n}$ is determined by the coproduct

$\mathbb{C}\left[x_{i j}, \operatorname{det}\left(x_{i j}\right)^{-1}\right] \hookrightarrow \mathbb{C}\left[y_{i j}, \operatorname{det}\left(y_{i j}\right)^{-1}\right] \otimes \mathbb{C}\left[z_{i j}, \operatorname{det}\left(z_{i j}\right)^{-1}\right], \quad\left(x_{i j}\right)=\left(y_{i j}\right) \circ\left(z_{i j}\right)$.

The group law in $G=\operatorname{Spec} \mathbb{C}\left[e^{M t}, \operatorname{det}\left(e^{-M t}\right)\right]$ is defined by the induced coproduct in the quotient algebra $\mathbb{C}\left[x_{i j}, \operatorname{det}\left(x_{i j}\right)^{-1}\right] \longrightarrow \mathbb{C}\left[e^{M t}, \operatorname{det}\left(e^{-M t}\right)\right]$, that is,

$$
\begin{aligned}
\mathbb{C}\left[e^{M t}, \operatorname{det}\left(e^{-M t}\right)\right] & \hookrightarrow \mathbb{C}\left[e^{M u}, \operatorname{det}\left(e^{-M u}\right)\right] \otimes \mathbb{C}\left[e^{M v}, \operatorname{det}\left(e^{-M v}\right)\right], \\
e^{M t} & =e^{M u} \circ e^{M v}=e^{M(u+v)} .
\end{aligned}
$$

In other words, this coproduct takes each function $f(t)$ into $f(u+v)$.

As shown in the proof of Lemma 4.6, the coordinate ring of $G$ has the form

$$
\mathbb{C}\left[\delta t, e^{\mu_{1} t}, \ldots, e^{\mu_{r} t}, e^{-\sum \mu_{j} t}\right] .
$$

Recall that the functions $t, e^{\mu_{1} t}, \ldots, e^{\mu_{r} t}$ are algebraically independent (Remark 4.4). With the coproduct $f(t) \mapsto f(u+v)$, the Hopf algebra

$$
\mathbb{C}\left[\delta t, e^{\mu_{1} t}, \ldots, e^{\mu_{r} t}, e^{-\sum \mu_{j} t}\right]
$$

has an obvious decomposition as a tensor product of Hopf algebras:

$$
\mathbb{C}\left[\delta t, e^{\mu_{1} t}, \ldots, e^{\mu_{r} t}, e^{-\sum \mu_{j} t}\right]=\mathbb{C}[\delta t] \otimes \mathbb{C}\left[e^{\mu_{1} t}, e^{-\mu_{1} t}\right] \otimes \cdots \otimes \mathbb{C}\left[e^{\mu_{r} t}, e^{-\mu_{r} t}\right],
$$

hence $G=\mathbb{G}_{a}^{\delta} \times \mathbb{G}_{m} \times . r . \times \mathbb{G}_{m}$.

Remark 4.9. By Theorem 3.4 a, there exists a $G$-invariant dense open set $U$ in $\mathbb{C}^{n}$ such that the orbits of $G$ in $U$ coincide with the minimal tangent subvarieties of $D$ in $U$. The isotropy subgroup of any point of $U$ is the identity subgroup. Let us give a (summarized) proof of this fact: Every (flat) family of subgroups of $G=\mathbb{G}_{m}^{r} \times \mathbb{G}_{a}^{\delta}$ is a constant family, hence all the points of $U$ have the same isotropy subgroup (generically); since $G$ acts faithfully on $U$, we conclude that such a subgroup is the identity. Therefore, the orbits of $G$ in $U$ have the same dimension as $G$, that is to say:

Minimal tangent subvarieties of a linear vector field $D=\sum \lambda_{i j} z_{j} \frac{\partial}{\partial z_{i}}$ on $\mathbb{C}^{n}$ have dimension $r+\delta$ (generically). 
By $3.4 \mathrm{~b}$, the field of rational functions on the quotient variety $Z=U / G$ coincides with the field of rational functions on $\mathbb{C}^{n}$ which are first integrals of $D$. Since $\operatorname{dim} Z=\operatorname{dim} U-\operatorname{dim} G=n-r-\delta$, we conclude that:

The field of all rational functions on $\mathbb{C}^{n}$ which are first integrals of a linear vector field $D=\sum \lambda_{i j} z_{j} \frac{\partial}{\partial z_{i}}$ has transcendence degree $n-r-\delta$.

This field may be computed by methods of Linear Algebra [3].

4.10. Let $\mathbb{P}_{n}$ be the $n$-dimensional projective space and let $\pi: \mathbb{C}^{n+1} \longrightarrow \mathbb{P}_{n}$ be the natural projection. It is well known that any vector field $D$ on $\mathbb{P}_{n}$ is the projection by $\pi$ of some linear vector field $D_{0}=\sum \lambda_{i j} z_{j} \frac{\partial}{\partial z_{i}}$ on $\mathbb{C}^{n+1}$. Moreover, such a linear field is unique up to the addition of a vector field proportional to $\sum z_{i} \frac{\partial}{\partial z_{i}}$. These facts follow readily from the standard exact sequence

$$
0 \longrightarrow \mathcal{O}_{\mathbb{P}_{n}} \longrightarrow \operatorname{Hom}_{\mathcal{O}_{\mathbb{P}_{n}}}\left(\mathcal{O}_{\mathbb{P}_{n}}(-1), \mathcal{O}_{\mathbb{P}_{n}}^{n+1}\right) \longrightarrow \mathcal{D}_{\mathbb{P}_{n}} \longrightarrow 0
$$

where $\mathcal{O}_{\mathbb{P}_{n}}(-1)$ is the sheaf of sections of the tautological line bundle on $\mathbb{P}_{n}, \mathcal{D}_{\mathbb{P}_{n}}$ is the sheaf of vector fields on $\mathbb{P}_{n}$ and $\operatorname{Hom}_{\mathcal{O}_{\mathbb{P}_{n}}}\left(\mathcal{O}_{\mathbb{P}_{n}}(-1), \mathcal{O}_{\mathbb{P}_{n}}^{n+1}\right)$ is the sheaf of $\pi$-projectable vector fields.

Theorem 4.11. Let $D=\pi_{*}\left(\sum \lambda_{i j} z_{i} \frac{\partial}{\partial z_{i}}\right)$ be a vector field on $\mathbb{P}_{n}$. Its associated group is

$$
G=\mathbb{G}_{m}^{s} \times \mathbb{G}_{a}^{\delta}
$$

where $s$ stands for the dimension of the minimal $\mathbb{Q}$-affine subspace of $\mathbb{C}$ containing all the eigenvalues of the matrix $\left(\lambda_{i j}\right)$, and $\delta=0$ when such a matrix is diagonalizable and $\delta=1$ otherwise.

Proof. Let $D_{0}=\sum \lambda_{i j} z_{i} \frac{\partial}{\partial z_{i}}$. After the addition of a vector field proportional to $\sum z_{i} \frac{\partial}{\partial z_{i}}$, we may assume that the matrix $M=\left(\lambda_{i j}\right)$ has the eigenvalue 0 . In such case the minimal $\mathbb{Q}$-affine subspace containing the eigenvalues of $\left(\lambda_{i j}\right)$ is a $\mathbb{Q}$-vector space. By Theorem 4.8 the group associated to $D_{0}$ is

$$
G_{0}=\mathbb{G}_{m}^{s} \times \mathbb{G}_{a}^{\delta} .
$$

Let $0 \neq v \in \mathbb{C}^{n+1}$ be an eigenvector of $M$ of eigenvalue 0 . Note that $v$ is a fixed point of the infinitesimal automorphism $\tau_{\varepsilon}^{0}$ (corresponding to $D_{0}$ ) since $\tau_{\varepsilon}^{0}(v)=e^{\varepsilon M}(v)=(I d+\varepsilon M)(v)=v$.

Let $H_{v}$ be the stabilizer of $v$, which is a closed algebraic subgroup of $G l_{n+1}$. The functor of points of $H_{v}$ is

$$
H_{v}^{\bullet}(T)=\left\{g \in G l_{n+1}^{\bullet}(T): g(v)=v\right\} .
$$

We have that $H_{v}$ is a tangent subvariety of $\left(G l_{n+1}, \tilde{D}_{0}\right)$, since

$$
g \in H_{v}^{\bullet}(T) \Rightarrow \tilde{\tau}_{\varepsilon}^{0}(g)=\tau_{\varepsilon}^{0} \circ g \in H_{v}^{\bullet}(T) .
$$

Since $G_{0}$ is minimal we obtain that $G_{0} \subseteq H_{v}$.

Analogously, denoting by $H_{p}$ the stabilizer of $p=\pi(v) \in \mathbb{P}_{n}$ with respect to the action of $P G l_{n+1}=$ Aut $\mathbb{P}_{n}$, we may prove that $H_{p}$ is a tangent subvariety of $\left(P G l_{n+1}, \tilde{D}\right)$ and then $G \subseteq H_{p}$.

Now, it is immediate that the natural epimorphism $G l_{n+1} \rightarrow P G l_{n+1}$ induces a differential isomorphism $\left(H_{v}, \tilde{D}_{0}\right) \longrightarrow\left(H_{p}, \tilde{D}\right)$. Via this isomorphism, we conclude that $G_{0}=G$. 
Remark 4.12. The same arguments used in Remark 4.9 show that:

Minimal tangent subvarieties of a vector field $D$ on $\mathbb{P}_{n}$ have dimension $s+\delta$ (generically).

The field of all rational functions on $\mathbb{P}_{n}$ which are first integrals of a vector field has transcendence degree $n-s-\delta$. (The case $n=2$ is well known; see [7, pp. 12-16.)

\section{A counterexample}

Without the hypothesis of $X$ being complete, it does not follow the existence, for any vector field $D$ on $X$, of a dense open set $U$ and a projection $\varphi: U \rightarrow Z$ whose fibres are the minimal tangent subvarieties of $D$ in $U$.

As a counterexample, let us consider the field

$$
D=z_{1} z_{4} \frac{\partial}{\partial z_{4}}+z_{2} z_{5} \frac{\partial}{\partial z_{5}}+z_{3} z_{6} \frac{\partial}{\partial z_{6}}
$$

on $X=\mathbb{C}^{6}$. It is clear that $D$ is tangent to any 3-plane $z_{1}=\lambda_{1}, z_{2}=\lambda_{2}, z_{3}=\lambda_{3}$. On these planes $D$ is a linear vector field with associated group $\mathbb{G}_{m}^{r}$, where $r$ is the dimension of the $\mathbb{Q}$-vector space $\mathbb{Q} \lambda_{1}+\mathbb{Q} \lambda_{2}+\mathbb{Q} \lambda_{3}$ (see Theorem 4.8). In each 3 -plane, the minimal tangent subvarieties generically have dimension $r$ (Remark 4.9). Now, points $\left(\lambda_{1}, \lambda_{2}, \lambda_{3}\right) \in \mathbb{C}^{3}$ with $r=3$ form a dense set in $\mathbb{C}^{3}$ (and so do points with $r=2$ ). Therefore, minimal tangent subvarieties of dimension 3 , as well as those of dimension 2 , form a dense subset of $\mathbb{C}^{6}$. This fact prevents the existence of such projection $\varphi: U \rightarrow Z$, due to the semicontinuity of the dimension of the fibres of any algebraic morphism.

\section{The CASe of Positive CharaCteristic}

In this section, $k$ denotes a field of characteristic $p>0$. Let $\mathbb{G}_{a}=\operatorname{Spec} k[t]$ be the additive line. The subscheme $G:=\operatorname{Spec} k[t] /\left(t^{p}\right)$ is a finite infinitesimal subgroup of $\mathbb{G}_{a}$.

Proposition 6.1. Let $X$ be a $k$-scheme. Each vector field $D$ on $X$ defines an action $\mu: G \times_{k} X \rightarrow X$,

$$
\mu^{*}: \mathcal{O}_{X} \rightarrow \mathcal{O}_{X}[t] /\left(t^{p}\right), \quad \mu^{*}(f)=\sum_{r=0}^{p-1} \frac{1}{r !} D^{r}(f) t^{r} .
$$
$X$.

Conversely, any action $\mu: G \times_{k} X \rightarrow X$ is defined by a unique vector field $D$ on

The proof of Proposition 6.1 is a simple exercise.

Let $D$ be a vector field on $X$ and let $\mu: G \times_{k} X \rightarrow X$ be the corresponding action. If $Y$ is a tangent subscheme of $(X, D)$, then the corresponding action $G \times_{k} Y \rightarrow Y$ is the restriction to $Y$ of the action of $G$ on $X$. Therefore, a closed subscheme $Y$ of $X$ is tangent to $D$ if and only if it is $G$-invariant. We conclude that the minimal tangent subscheme passing through a closed point $x$ is the orbit $G \cdot x=$ scheme-theoretic image of $G \times x \subseteq G \times X \stackrel{\mu}{\longrightarrow} X$, that is to say, $G \cdot x$ is the closed subscheme of $X$ defined by the ideal of all functions $f$ such that $f(x)=D f(x)=\cdots=D^{p-1} f(x)=0$. Note that each orbit has a unique point, but it is not a reduced scheme in general. 


\section{Appendix A. Quotients By Algebraic groups}

Theorem A.1 (Rosenlicht [11]). Let $\mu: G \times_{k} X \rightarrow X$ be an action of an affine algebraic group $G$ on an integral quasi-projective variety $X$. There exists a $G-$ invariant dense open subset $U \subseteq X$ such that the geometric quotient $U \rightarrow U / G$ exists.

The purpose of this Appendix is to extend Rosenlicht's result to the case of an algebraic group $G$ non-necessarily affine.

Quotients by abelian varieties. Let $\mu: A \times_{k} X \rightarrow X$ be an action of an abelian variety $A$ on an integral quasi-projective variety $X$.

Lemma A.2. The action $\mu: A \times_{k} X \rightarrow X,(a, x) \mapsto a \cdot x$, and the morphism $\phi: A \times_{k} X \rightarrow X \times_{k} X,(a, x) \mapsto(a \cdot x, x)$, are projective morphisms.

Proof. The isomorphism $\varphi: A \times X \rightarrow A \times X,(a, x) \mapsto(a, a \cdot x)$, makes commutative the triangle

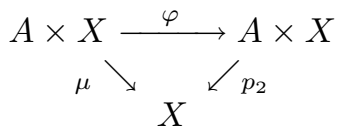

Since any abelian variety is projective, the map $p_{2}: A \times X \rightarrow X$ is a projective morphism and then the above commutative triangle implies that $\mu: A \times X \rightarrow X$ is also a projective morphism. Finally, $\phi=\mu \times p_{2}$ is a projective morphism because $\mu$ and $p_{2}$ are also.

Let $R$ be the image of the map $\phi: A \times_{k} X \rightarrow X \times_{k} X,(a, x) \mapsto(a \cdot x, x)$, that is to say, $R$ is the equivalence relation defined by the action of $A$ over $X$. By the previous lemma, $R$ is a closed subset of $X \times_{k} X$. We shall consider $R$ as a closed subscheme of $X \times_{k} X$ with the reduced structure.

Lemma A.3. The projection $p_{1}: R \rightarrow X$ is a projective morphism.

Proof. Since $\phi: A \times X \rightarrow R$ is surjective and the composition morphism $\mu=$ $p_{1} \circ \phi: A \times X \rightarrow R \rightarrow X$ is proper, it is easy to check that the valuative criterion of properness ([6], II, Th. 4.7) holds for the morphism $p_{1}: R \rightarrow X$.

Moreover, $R \subseteq X \times X \stackrel{p_{1}}{\longrightarrow} X$ is a quasi-projective morphism, hence we conclude that $p_{1}: R \rightarrow X$ is a projective morphism.

Given an $A$-stable open subset $U$ of $X$, we write $R_{U}:=p_{1}^{-1}(U)$, i.e., $R_{U}$ is the equivalence relation defined by the action of $A$ over $U$.

Lemma A.4. There exists an A-invariant dense open subset $U$ of $X$ such that $p_{1}: R_{U} \rightarrow U$ is a flat morphism.

Proof. By the semicontinuity character of the Hilbert polynomial of the fibres of a projective morphism $p_{1}: R \rightarrow X$, we have that the subset $U$ of all points $x \in X$, whose fibre $p_{1}^{-1}(x)$ has the same Hilbert polynomial than the fibre of the generic point, is a dense open subset of $X$. This open subset $U$ is invariant by the action of $A$ over $X$, since the projection $p_{1}: R \rightarrow X$ is an $A$-equivariant morphism (the action of $A$ over $R$ is defined by the formula $\left.a \cdot\left(x_{1}, x_{2}\right)=\left(a \cdot x_{1}, x_{2}\right)\right)$.

Finally, since the fibres of $p_{1}: R_{U} \rightarrow U$ have the same Hilbert polynomial, we conclude (see [6], III, Th. 9.9) that $p_{1}: R_{U} \rightarrow U$ is a flat morphism. 
Theorem A.5. Let $\mu: A \times_{k} X \rightarrow X$ be an action of an abelian variety $A$ on an integral quasi-projective variety $X$. There exists an $A$-invariant dense open subset $U$ of $X$ such that the geometric quotient $\pi: U \rightarrow U / A$ exists.

Proof. By the previous lemmas, there exists an $A$-invariant dense open subset $U$ of $X$ such that $p_{1}: R_{U} \rightarrow U$ is a projective flat morphism. By a theorem of Grothendieck ([5, V, Th. 7.1), there exists the quotient map $\pi: U \rightarrow U / A$.

\section{The general case.}

Theorem A.6. Let $\mu: G \times_{k} X \rightarrow X$ be an action of a connected smooth group $G$ on an integral quasi-projective variety $X$. There exists a $G$-invariant dense open subset $U \subseteq X$ such that the geometric quotient $U \rightarrow U / G$ exists.

Proof. By the structure theorem of algebraic groups [12, there exists a normal affine subgroup $G_{0}$ of $G$ such that $A=G / G_{0}$ is an abelian variety. By Rosenlicht's result, there exists a $G_{0}$-invariant dense open subset $U_{0} \subseteq X$ such that the geometric quotient $\pi: U_{0} \rightarrow U_{0} / G_{0}$ exists. Taking $G \cdot U_{0}$ instead of $U_{0}$, we may assume that $U_{0}$ is $G$-invariant. By Theorem A.5 there exists an $A$-invariant dense open subset $V$ in $U_{0} / G_{0}$ such that the geometric quotient $V \rightarrow V / A$ exists. Then $U:=\pi^{-1} V$ is the desired open set, since $U / G=\left(U / G_{0}\right) / A=V / A$.

\section{REFERENCES}

[1] C. Chevalley, Théorie des Groupes de Lie, Hermann, Paris (1968).

[2] M. Demazure \& P. Gabriel Groupes Algébriques, Masson \& North-Holland, Amsterdam (1970). MR 46:1800

[3] R. FARO \& J.B. SANChO, Rational first integrals of linear differential equations, (preprint).

[4] A. Grothendieck, Fondements de la Géométrie Algébrique, Séminaire Bourbaki 1957-62, Secrétariat Math., Paris (1962). MR26:3566

[5] A. Grothendieck \& M. Demazure, Schémas en Groupes I, Lecture Notes in Math. 151, exp. V, Springer-Verlag, Heidelberg (1970). MR 43:223a

[6] R. Hartshorne, Algebraic Geometry, Grad. Texts in Math. 52, Springer-Verlag, New York (1977). MR 57:3116

[7] J.P. Jounanolou, Equations de Pfaff algébriques, Lecture Notes in Math. 708, SpringerVerlag, New York (1979). MR81k:14008

[8] A.R. Magid, Lectures on Differential Galois Theory, Univ. Lecture Series 7, Amer. Math. Soc., Providence, R.I. (1994). MR95j:12008

[9] H. Matsumura \& F. Oort, Representability of Group Functors, and Automorphisms of Algebraic Schemes, Inventiones Math. 4 (1967) 1-25. MR.36:181

[10] M. VAN DER PUT \& M.F. Singer, Galois Theory of Linear Differential Equations, Grundlehren der mathematischen Wissenschaften, vol. 328, Springer-Verlag (2003).

[11] M. Rosenlicht, A remark on Quotient Spaces, Ana. da Acad. Brasiliera de Ciencias 35 (1963) 25-28. MR 30:2009

[12] M. Rosenlicht, Some Basic Theorems on Algebraic Groups, Amer. J. Math. 78 (1956) 401-443. MF 18:514a

[13] C. Sancho, Grupos Algebraicos y Teoria de Invariantes, Univ. Autónoma de Mexico (2002). MR 2003i:20085

Departamento de Matematicas, Universidad de Extremadura, Av. de Elvas s/n, BadaJOZ 06071, SPAIN

E-mail address: jsancho@unex.es 